\title{
CMOS Compatible Hyperspectral Optical Filters
}

\author{
Damiana Lerose ${ }^{1}$, Detlef Sommer ${ }^{1}$, Konrad Bach ${ }^{1}$, Daniel Gäbler ${ }^{1}$, Martin Sterger ${ }^{2}$ \\ ${ }^{1} X-F A B$ Semiconductor Foundries AG, Haarbergstr. 67, 99097 Erfurt, Germany,
}

detlef.sommer@xfab.com

${ }^{2} X-F A B$ Dresden GmbH \& Co KG, Grenzstrasse 28, 01109 Dresden, Germany

\begin{abstract}
Commonly, optical filters consist of pigments on glass or of many stapled interference layers. In both cases, they are not applied on CMOS devices within the fab line, as they are not CMOS-compatible. Consequently, further processing is needed and high additional costs are incurred. It is presented a method for producing tuneable optical filters with tuneable transmission spectra in a CMOS-line.The filters are constituted by a Fabry-Perot resonator formed by two Bragg mirrors separated by a patterned cavity. The filter response can be tuned by changing the geometric parameters of the patterning, and consequently the cavity effective refractive index. In this way, many different filters can be produced at once on a single chip, allowing multichanneling. The filter has been designed, produced, and characterized. The results for a chip with 24 filters are presented.
\end{abstract}

Key words: Hyperspectral Filters, CMOS filter, spectroscopy, Fabry-Perot resonator, Bragg mirrors

\section{Introduction}

Opto-electronic sensors are used in diverse fields of applications for consumer, industrial and scientific products. Several of these applications have the need for the detection of specific wavelengths. Such requirements include the possibility of changing the filter response within a precise wavelength range. There is a high interest in developing optical filters which can be realized with an inexpensive process.

On the other hand CMOS is widely used for light sensitive sensors. It was successful for image sensors as used in mobile phones and is also used for several other sensor applications like ambient light sensors for controlling the display brightness, proximity sensors and optocouplers. Using CMOS for realizing precise, inexpensive and high reliable filters would provide a great benefit.

This article describes a CMOS-compatible process fulfilling such challenging requirements as (i) determining the filter response wavelength range by few process-independent parameters,

(ii) integrated filters with different optical responses on the same chip allowing multichanneling in view of precise spectral analysis.

(iii) integrated filter fulfilling automotivequalifying requirements of stability and reliability.

\section{Principle}

The filter consists of a stack of CMOScompatible materials and can be described as a Fabry-Perot interferometer which is formed by two similar Bragg mirrors separated by a layer in the middle. Light is reflected between the two mirrors so that constructive or destructive interference occurs and the desired wavelengths are filtered. The used mirrors are formed by a stack of layers constituted alternately by two media with different refractive index, e.g. silicon and silicon oxide. The thickness of the layers is usually set such that 
the interference at the layer interface is constructive or destructive in the same way, so that a spectral region with high reflectivity is obtained. This is achieved when the layers have the same optical thickness dopt $=\mathrm{n}-$ dphys, where $n$ is the refractive index of the layer and dphys the physical layer thickness.

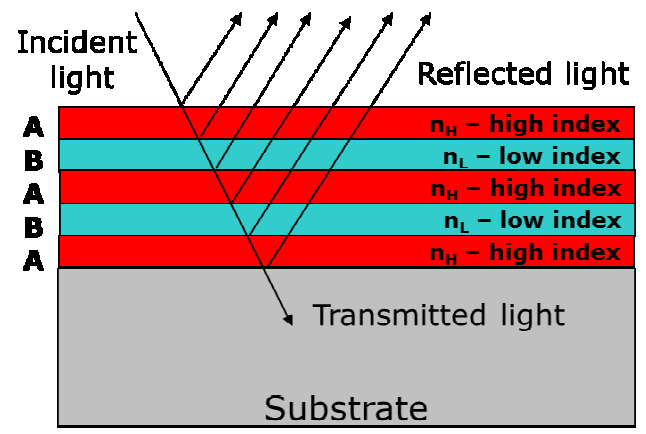

Fig. 1. Fabry-Perot interferometer formed by two similar Bragg mirrors separated by a layer in the middle.

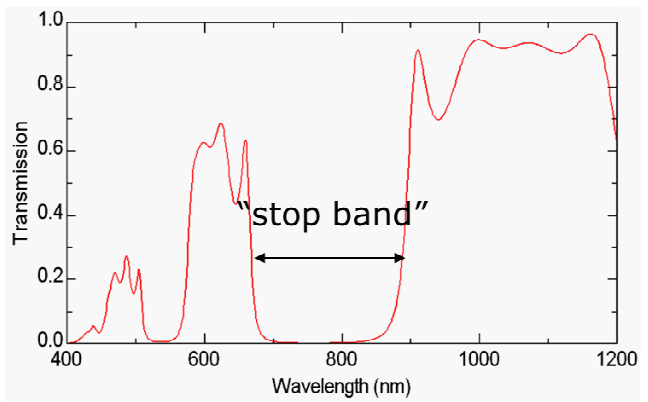

Fig. 2. Stop band generated by Brack mirrors.

If the layer between the two mirrors (cavity) is characterized by different thickness and/or refractive index than the mirrors, a transmission peak appears within the stop band. Its wavelength $\lambda$ peak is given by

$$
\lambda \text { peak }=4 \square \mathrm{n}-\text { dphys (1) }
$$

or multiples thereof. The peak wavelength can be shifted by changing the cavity thickness and/or its refractive index. While changing its thickness appears to be the most obvious solution [2], it is not convenient from a production point of view.

Contrary, considering the cavity layer as an effective medium, a change in its patterning causes a modification of its refractive index, which determines the shift of the peak wavelength. The change in the refractive index can be achieved with only one additional mask and offers a way for shifting the peak wavelength which is highly controllable, easier to integrate on chip and more robust towards process tolerances.

Considering a cavity constituted by silicon which is structured and refilled with $\mathrm{Si}$ oxide then the effective medium approximation (EMA) theories state that it is possible to consider such layer as constituted by one unique material, namely the "effective medium". Its physical properties can be calculated from the ones of the constituent materials by using different approaches and formula. Of particular interest for this purpose is the calculation of the effective refractive index, which strongly depends on the volume percentages of the two used materials. The Maxwell-Garnett formula [1] is widely used for this goal. The calculated effective refractive index $n_{\text {eff }}$ must substitute $n$ in Eq. (1) for predicting the peak wavelength of the filter. This is the key point: the peak wavelength changes when varying $n_{\text {eff }}$, which depends on the volume ratio of $\mathrm{Si}$ oxide to $\mathrm{Si}$ in the cavity layer. Consequently, by changing the pattern of this layer, one obtains filters which have a response targeted on different wavelengths. The difference in the volume ratio of neighboring filters can be such that a peak wavelength difference as small as few nanometers can be achieved, allowing hyperspectral detection. The EMA is valid as long as the size of the structures of the inclusive medium is smaller than the considered light wavelength.

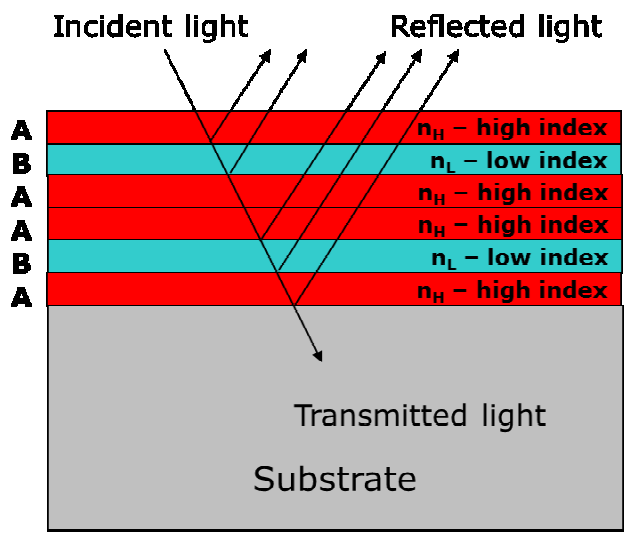

Fig. 3. Cavity within the mirrors with different refractive index. 


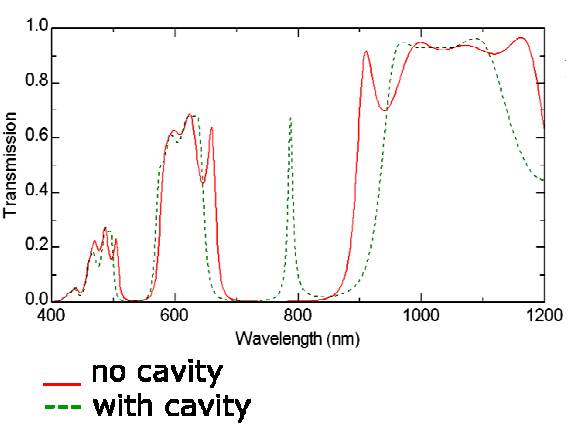

Fig. 4. Transmission peak within the stop band

This approach was tested between 800 and $1000 \mathrm{~nm}$. For applications in different spectral regions other CMOS-compatible materials might be more suitable, taking into account their absorption coefficient for those wavelengths and whether the difference in their refractive indexes is big enough.

\section{Calculation and design}

Filter simulations have been performed in order to determine the most suitable physical parameters for its production, to design the filter, and to predict the filter optical properties.

For reliable results, the exact optical parameters of the used materials and the precise filter design have been uploaded. The optical parameters are represented by a model which allows for each material the calculation of the refractive index in its real and complex parts as a function of the wavelength.

Different deposition parameters have been tested and their influence on the optical properties of the layer has been studied. The compatibility of the process steps with the overall production flow has been taken into account as well. As the filter is deposited on top of a CMOS device, the filter design must consider not only the stack of the Bragg mirrors, but also the layers below them which result from the previous CMOS processing.

This insulating oxide stack influences the filter response, as it acts as an additional irregular interferometer, adding undesired ripples in the transmission spectrum. Moreover, other layers, like the passivation, are present. The impact of all these additional unavoidable layers has been studied by calculations which also allow tuning the response of the Fabry-Perot filter accordingly, so that the whole system gives the desired optical response.

\section{Realization}

Only existing or compatible CMOS processes can be used for the production of the filter. This limits the available materials, the layer thickness, the cavity patterning, and the thermal budget. Additionally, the wafer characteristics after CMOS processing prior to filter deposition (e.g. wafer bow) needs to be considered for determining any impact on the filter quality too.

The filter is deposited on top of the CMOS device, which makes it compatible with any technology node or layout. The changes introduced in the process flow for the production of the filter include the modification of existing processes as well as new steps, where the in-line process parameters like layer thickness, wafer bow, pattern quality have been monitored.

The production of the filter does not influence the standard CMOS process, as the filter stack is deposited on top of the device. Nevertheless, some few changes must be undertaken to allow the integration of the filter in the chip [5]. The oxide covering the last metal layer must be planarized to be able to deposit the filter layers; the passivation is deposited on top of the filter instead of on top of the last metal. Therefore the etch recipe of the pads must be tuned so that the metal can be reached through a thicker stack.

Additionally, a process was introduced for the deposition of the filter layers with particular requirements on temperature and homogeneity and finally patterning the cavity layer.

\section{Results}

The filter response is obtained dividing the sensitivity of identical photodiodes on the same chip with and without ("reference") optical filter.

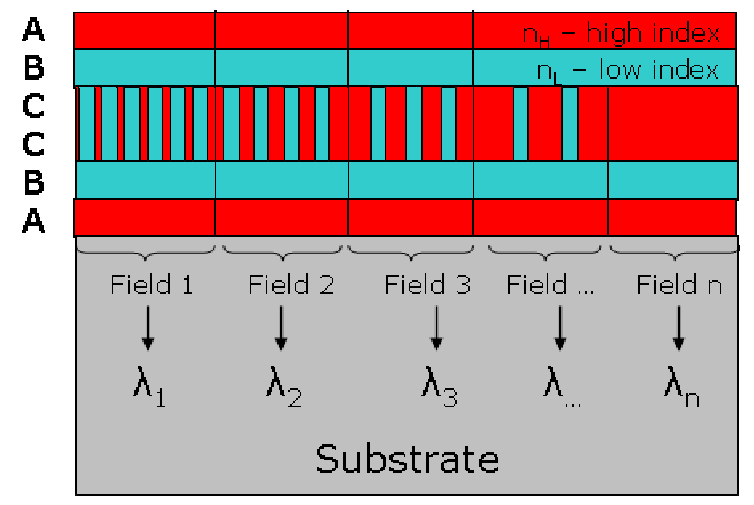

Fig. 5. Schematic representation of the filter in cross section on top of a simplified CMOS device. The different patterning of the cavity layer can be tuned to achieve the desired transmission peak wavelength(s). 
An example of the achieved results is shown in Fig. 6. The different intensity of the transmission peaks is due to the interference of the filter stack with the other layers. Such interference does not add significant oscillations to the filter response (position and width of the peak are unchanged, as the transmission peak is sharper than the oscillations themselves), but modulates the peak intensity. The angledependence of the filter has been addressed in various simulations and it results in a blue-shift of the transmission peak of about $10 \mathrm{~nm}$ if incident angles of $5^{\circ}$ and $25^{\circ}$ are compared. A systematic investigation of this dependence was not possible due to limitations in the measurement set-up.

\section{Conclusion}

A CMOS-integrated optical filter which consists of a Fabry-Perot resonator formed by two Bragg mirrors has been presented. The filter response is characterized by a transmission peak within a low-transmission region, whose spectral range can be freely set choosing the filter design and the filter materials. The transmission peak wavelength can be geometrically tuned within the low-transmission region by changing the pattern of the layer between the two mirrors as such wavelength depends on the volume percentage of the materials in this layer. A total of 24 different filters on the same chip have been achieved with one process step. The filter processing and its production is completely CMOS compatible. The production of the filter only requires few additional process steps. Sharp peaks and hyperspectral multichanneling in the near-infrared region show that the filter response does not significantly depend on temperature in the considered range. This filter is the first example of a CMOS integrated hyperspectral filter which allows multichanneling based only on layout and processed completely within the CMOS-line with standard tools.

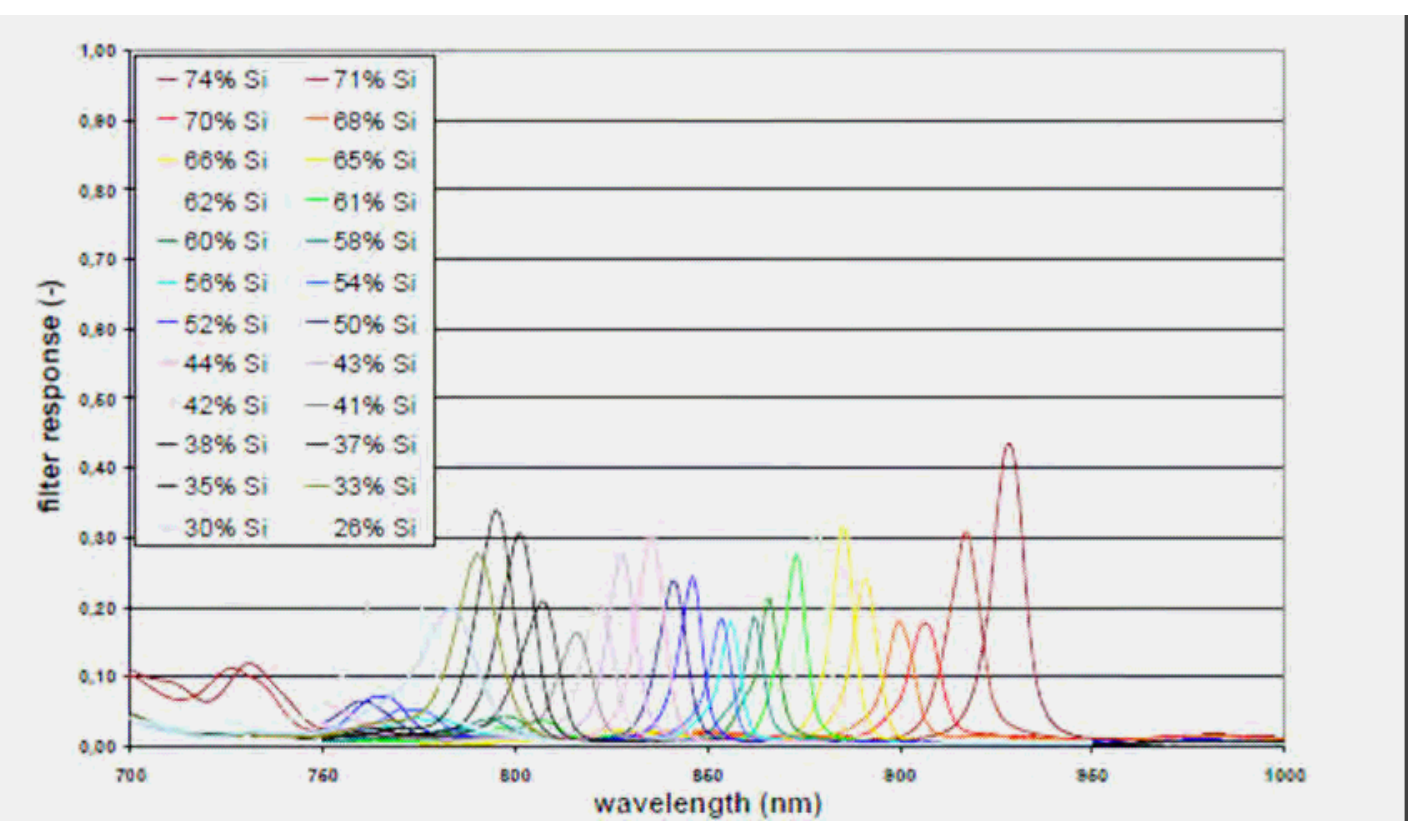

Fig. 6. Filter response measured on one single chip which represents the described CMOS-integrated filter. The filter consists of a cavity layer with 24 different patterns in correspondence with 24 photodiodes. The patterns are characterized by different volume percentages of Si in the cavity layer and have been set using simulations and confirmed by inline measurements of the feature areas. Such percentages are shown in the legend. The patterning of the cavity layer is the only difference among the filters. Therefore, all 24 filter responses are achieved with the same process steps. 


\section{References}

[1] L.C. Kimerling, D. Ahn, A. B. Apsel, M. Beals, D. Carothers, Y.-K. Chen, T. Conway, D. M. Gill, M. Grove, C.-Y. Hong, M. Lipson, J. Liu, J. Michel, D. Pan, S. S.Patel, A. T. Pomerene, M. Rasras, D. K. Sparacin, K.-Y.Tu, A. E. White, C. W. Wong, Electronic-photonic integrated circuits on the CMOS platform, Proc. SPIE 6125, 612502 (2006).

[2] J. H. Correia, G. De Graaf, M. Bartek, R. F. Wolffenbuttel, A CMOS optical microspectrometer with light-to-frequency converter, bus interface, and straylight compensation, IEEE Transactions on Instrumentation and Measurement, 6, 1530-1537 (2002).

[3] J. C. M. Garnett, Colors in metal glasses and metal films, Trans. Roy. Soc. 53, 385-420 (1904)

[4] Lerose, D., Gäbler, D., Junger, S., CMOSintegrated geometrically tunable optical filter, Advanced Semiconductor Manufacturing Conference (ASMC), 2012 23rd Annual SEMI, pp. 97-101, 15-17 May 2012;

[5] Gäbler and D. Lerose, CMOS-kompatibles Herstellungsverfahren zur Realisierung eines planaren hyperspektralen optischen Filters, Patent Application, German Office Number 10 , 2011111883.0 (2011) 